Pacific

Journal of

Mathematics

EXAMPLES OF BIREDUCIBLE DEHN FILLINGS

James A. Hoffman and Daniel Matignon 


\title{
EXAMPLES OF BIREDUCIBLE DEHN FILLINGS
}

\author{
James A. Hoffman And Daniel Matignon
}

\begin{abstract}
If an irreducible manifold $M$ admits two Dehn fillings along distinct slopes each filling resulting in a reducible manifold, then we call these bireducible Dehn fillings. The first example of bireducible Dehn fillings is due to Gordon and Litherland. More recently, Eudave-Muñoz and Wu presented the first infinite family of manifolds which admit bireducible Dehn fillings. We present another infinite family of hyperbolic manifolds which admit bireducible Dehn fillings. The manifolds obtained by the fillings are always the connect sum of two lens spaces.
\end{abstract}

\section{Introduction.}

Let $M$ be an orientable 3-manifold with toroidal boundary $T$. Given a slope $r$ on $T$, the Dehn filling of $M$ along $r$, denoted by $M(r)$, is the manifold obtained by identifying $T$ with the boundary of a solid torus $V$ so that $r$ bounds a meridian disk in $V$.

In this paper, we are especially interested in those Dehn fillings which produce reducible manifolds. Recall that a manifold is reducible if it contains an essential 2-sphere, that is, a 2-sphere which does not bound a 3-ball. If an irreducible manifold $M$ admits two Dehn fillings along distinct slopes each filling resulting in a reducible manifold, then we call these bireducible Dehn fillings.

The first example of bireducible Dehn fillings is due to Gordon and Litherland [GLi]. More recently, Eudave-Muñoz and $\mathrm{Wu}[\mathbf{E W}]$ presented the first infinite family of manifolds with admit bireducible Dehn fillings. They show that for each $p \neq 0$ there is a hyperbolic manifold $M_{p}$ such that $M_{P}(\infty) \cong Q(2,-2) \# R P^{3}$ and $M_{P}(0) \cong Q(2 p,-2 p) \# R P^{3}$, where $Q(r, s)$ is the double branched cover of a Montesinos tangle $T[r, s]$.

We present another infinite family of hyperbolic manifolds which admit bireducible Dehn fillings, all with exactly one toroidal boundary component. They represent counterexamples to the generalization of the CablingConjecture [GS] since they are hyperbolic. Notice that examples with a single boundary component can be constructed from the examples given in $[\mathbf{E W}]$ (for more details, see the end of Section 4). 
Theorem 1. There exists an infinite family of hyperbolic manifolds which admit bireducible Dehn fillings. More precisely, there exist families of hyperbolic manifolds $M_{2}^{t}, M_{3}^{t}$ and $M_{4}^{t}$, parameterized by an integer $t$, such that:
a) $M_{2}^{t}(\infty) \cong L(-2,1) \# L(-4,1)$ and
$M_{2}^{t}(t) \cong L(2,1) \quad \# L\left(t^{2}-2 t+1, t-2\right)$ for $t \neq 0,1,2$,
b) $M_{3}^{t}(\infty) \cong L(-3,1) \# L(-3,1)$ and
$M_{3}^{t}(t) \cong L(3,1) \quad \# L\left(t^{2}-t+1, t-1\right)$ for $t \neq 0,1$,
c) $M_{4}^{t}(\infty) \cong L(-4,1) \# L(-2,1)$ and
$M_{4}^{t}(t) \cong L(4,1) \quad \# L\left(t^{2}+1, t\right)$ for $t \neq 0$.

Note that in each instance, the manifold resulting from the Dehn filling is the connect sum of two lens spaces. The lens space $L(p, q)$ is the manifold obtained by performing $p / q$-Dehn surgery on the unknot. The restrictions on the parameter $t$ are there to account for cases where either the resultant manifold is not reducible (i.e., one of the summands is $L(1, n) \cong S^{3}$ ), or one summand of the resultant manifold is not a lens space. The latter occurs in Case (a) with $t=1$. Here we get the summand $L(0,-1) \cong S^{2} \times S^{1}$. This case is also uninteresting since the manifold (before the Dehn filling) is reducible.

\section{Surgery instructions.}

In this section, we show how to construct a family of manifolds with one toroidal boundary component. Begin with the five component link $L \subset S^{3}$ shown in Figure 1 having components $A, B, C, D$ and $K$. The box labeled $t$ represents $t$ full twists. Positive values represent right-handed twists; and, negative values represent left-handed twists. For example, 
$=\sqrt{3}=200000$ and $\quad=-2 E=20006$

Define $M_{(a, b, c, d)}^{t}$ to be the manifold obtained by removing a regular neighborhood of $K$ and performing Dehn surgery on the components $A, B, C$ and $D$ along the respective slopes $a, b, c$ and $d$. The parameter $t$ represents the number of twists between the components $K$ and $C$ (as shown in Figure 1). In particular, we are interested in the families of manifolds $M_{(-1,-2,1,2)}^{t}, M_{(-1,-2,2,1)}^{t}, M_{(-2,-1,1,2)}^{t}$ and $M_{(-2,-1,2,1)}^{t}$ parametrized by the nonzero integer $t$.

Also, let $T=\partial M_{(a, b, c, d)}^{t}$ be the boundary torus. If $r$ is a slope in $T$, then define $M_{(a, b, c, d)}^{t}(r)$ to be the manifold obtained by performing a Dehn filling along $r$. 


\section{Bireducibility.}

Here we show that there are two slopes, namely $\infty=\frac{1}{0}$ and $t$, in the boundary of $M_{(a, b, c, d)}^{t}$ such that the Dehn fillings, $M_{(a, b, c, d)}^{t}(\infty)$ and $M_{(a, b, c, d)}^{t}(t)$, are both reducible manifolds. Moreover, the resulting summands are all lens spaces.

The following proofs use the link-calculus of 3-manifolds as described in Chapter $9 \mathrm{H}$ of $[\mathbf{R}]$. The proofs consist of a series of link diagrams with accompanying surgery coefficients. Each transition between diagrams is either an isotopy or a twisting about an unknotted component. In order to simplify the statements of the following claims, we will consider $L(1, n) \cong S^{3}$ and $L(0, \pm 1) \cong S^{2} \times S^{1}$ as lens spaces. This inclusion applies only to this section of the paper. Let us first consider the manifolds for which $a=-1$, $b=-2$.

\section{Claim 1. $M_{(-1,-2, c, d)}^{t}(t) \cong L\left(t^{2}+(1-c) t+1, t+1-c\right) \# L(d+2,1)$.}

Proof. We refer the reader to Figure 2. In the first transition, component $D$ is isotoped so that components $B$ and $D$ "pass through" component $A$ in like fashion. This is done to facilitate the twisting of component $A$ in the second transition.

In transition 2, we perform a single positive twist about component $A$. Since $A$ now has a trivial surgery coefficient, it is removed from the diagram. Note that the surgery coefficients increase for components $B$ and $D$ as they link component $A$. In transition 3, we perform a single positive twist about component $B$. Again, the component is removed as it has a trivial surgery coefficient; and, the surgery coefficients for components $C$ and $D$ are increased.

Transition 4 introduces a new component $F$ linking components $C$ and $K$, and gives it $-t-1$ twists (to unwind the twisting of components $C$ and $K$ ). Thus the surgery coefficient of $F$ is $\frac{1}{-t-1}$. This new component is temporary and simplifies the diagram for the next transition. Moreover, we achieve the desired effect that the surgery coefficient of $K$ is now -1 . At this point, component $C$ and $D$ have, respectively, surgery coefficients $c-t$ and $d+2$.

In transition 5 , we perform a single positive twist about component $K$, giving it a trivial surgery coefficient. We will keep $K$ in the diagram so that we may see how it lies in the resulting manifold. Note that the surgery coefficient of component $C$ is unchanged as it has linking number zero with component $K$. The surgery coefficient of $F$ is increased by the twist.

Transition 6 is an isotopy of component $C$. In transition 7 , components $D, F$ and $K$ are isotoped to facilitate a twisting about component $C$.

In transition 8 , we perform a single negative twist about component $C$. This is done so the coefficient of component $F$ once again represents $-t-1$ twists. Transitions 9 and 10 are isotopies of component $D$ in an attempt to 
separate components $C$ and $D$. In transition 9 , the lower arc of $D$ is flyped to the top of the diagram. Also, a lower loop of $D$ is untwisted. In transition 10 , component $D$ is pulled taut at the expense of twisting component $K$.

Transition 11 is an isotopy of components $F$ and $C$. Component $C$ is pulled down. We also localize component $F$ so that, in transition 12 , we may perform $t+1$ twists about $F$.

The final diagram shows us the knot $K$ with trivial surgery coefficient. If we disregard $K$, we see two unknotted and unlinked components. Each component, $C$ and $D$, represents a lens space summand (possibly $S^{3}$ or $\left.S^{2} \times S^{1}\right)$ of $M_{(-1,-2, c, d)}^{t}(t)$, thus proving the claim.

Next, consider the manifolds for which $a=-2$ and $b=-1$.

Claim 2. $M_{(-2,-1, c, d)}^{t}(t) \cong L\left(t^{2}-c t+1, t-c\right) \# L(d+1,1)$.

Proof. We refer the reader to Figure 3. The proof of this claim is nearly identical to the proof of Claim 1. Transition 1 is an isotopy of component $D$. In transition 2 , we perform a single positive twist on component $B$. This gives $B$ a trivial surgery coefficient; so it is removed. The surgery coefficient of components $A$ and $C$ increase to -1 and $c+1$, respectively. In transition 3 , we perform a single positive twist on component $A$. This gives $A$ a trivial surgery coefficient; so it too is removed. The surgery coefficients of components $C$ and $D$ each increase by 1 . The remaining transitions are identical to those in Claim 1, the only difference being the surgery coefficients of components $C$ and $D$. 


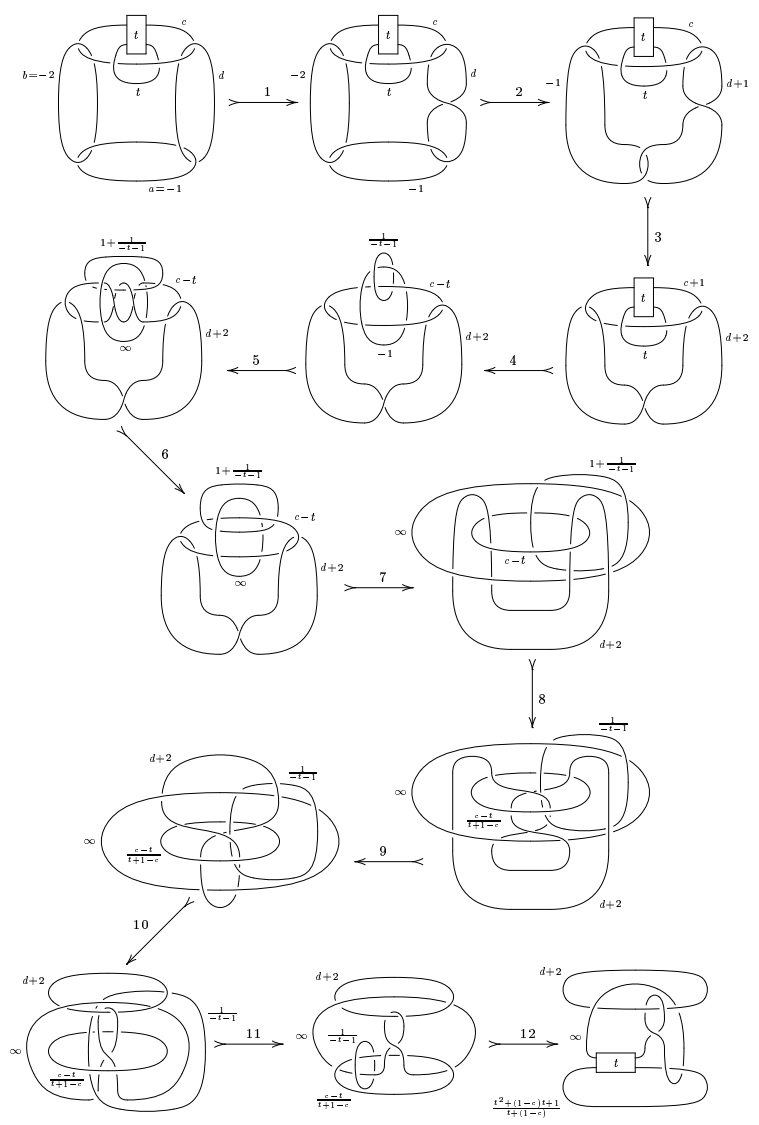

Figure 2. The equivalence of $M_{(-1,-2, c, d)}(t)$ and $L\left(t^{2}+(1-c) t+1, t+1-\right.$ c) \#L(d+2,1). 


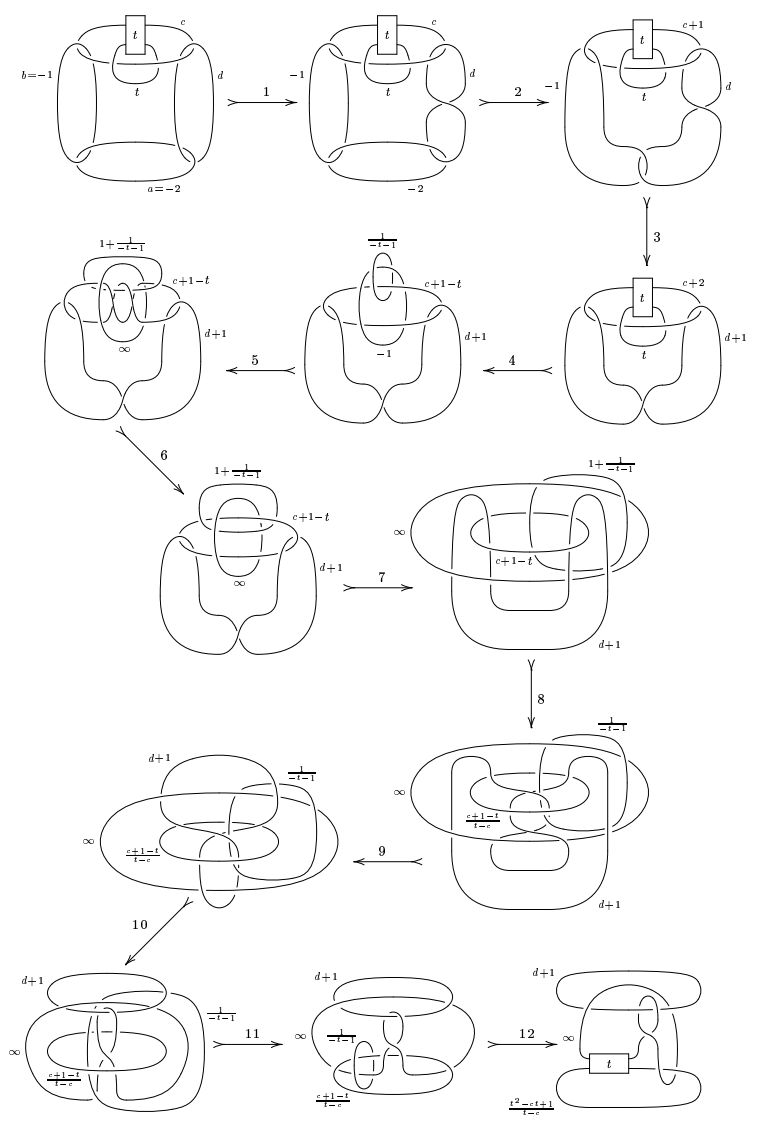

Figure 3. The equivalence of $M_{(-2,-1, c, d)}(t)$ and $L\left(t^{2}-c t+1, t-c\right) \# L(d+$ $1,1)$. 


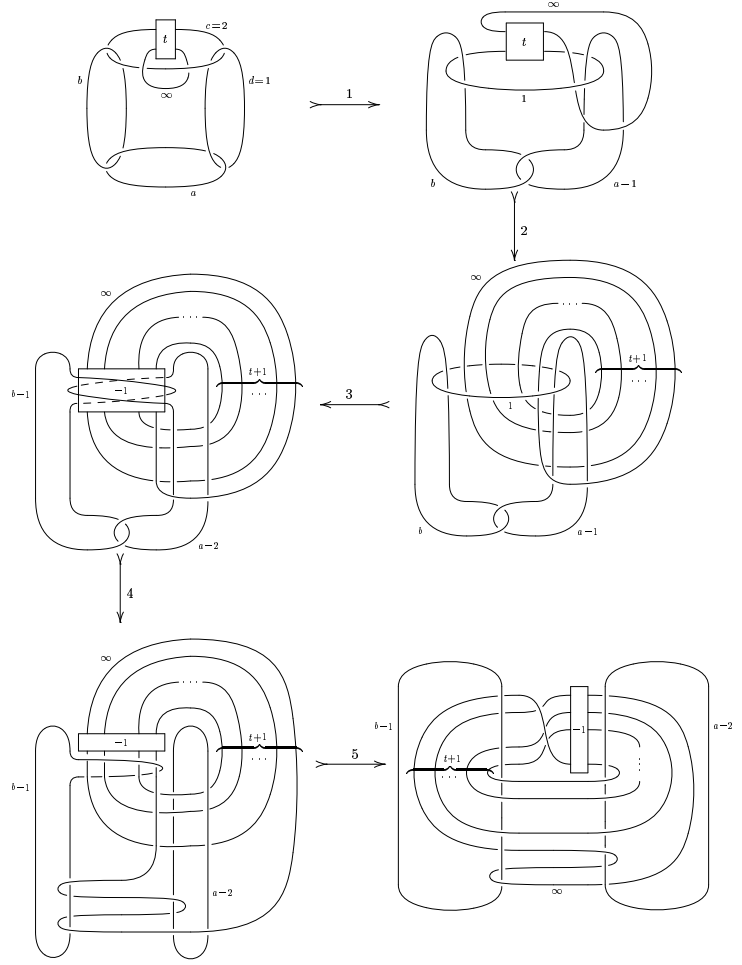

Figure 4. The equivalence of $M_{(a, b, 2,1)}^{t}(\infty)$ and $L(a-2,1) \# L(b-1,1)$ with $t>0$. 

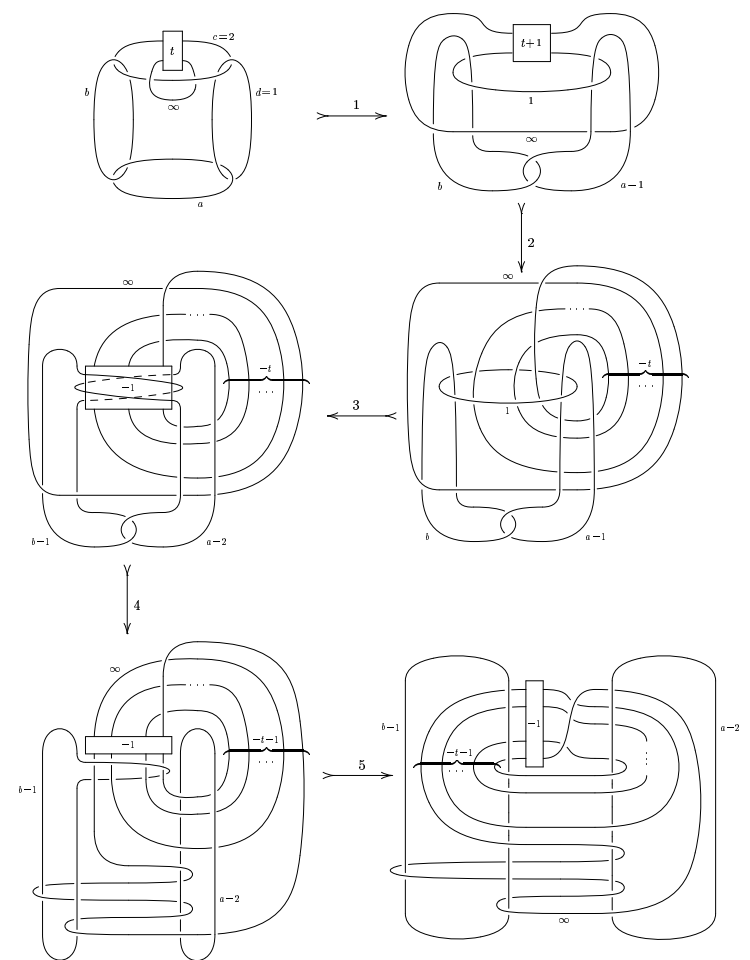

Figure 5. The equivalence of $M_{(a, b, 2,1)}^{t}(\infty)$ and $L(a-2,1) \# L(b-1,1)$ with $t<0$. 


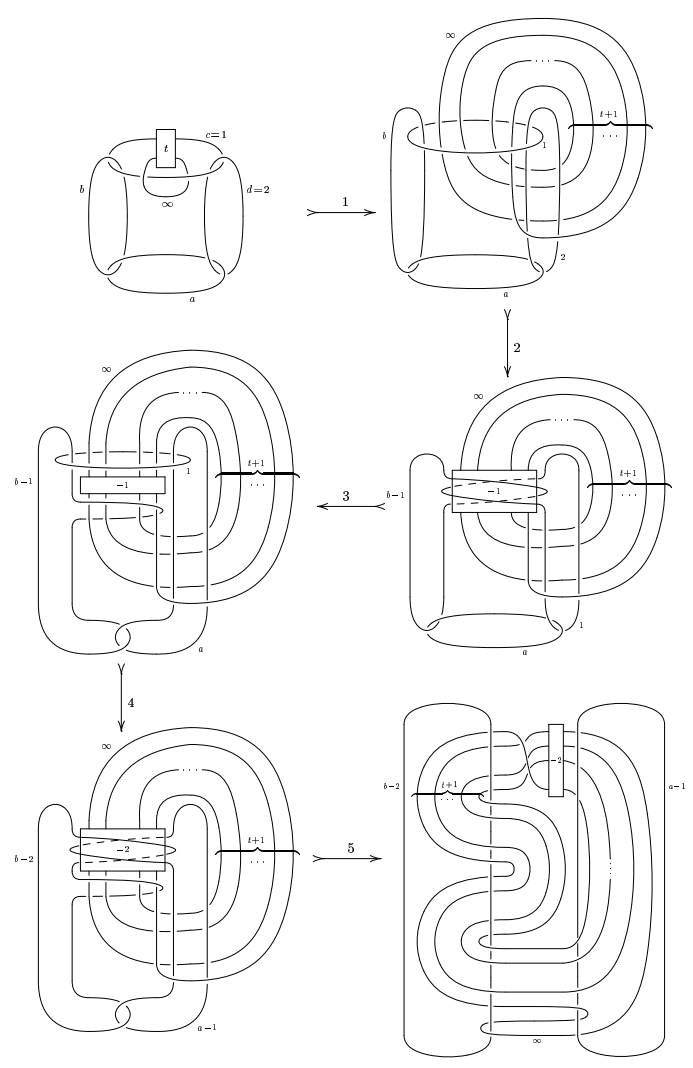

Figure 6. The equivalence of $M_{(a, b, 1,2)}^{t}(\infty)$ and $L(a-1,1) \# L(b-2,1)$ with $t>0$. 


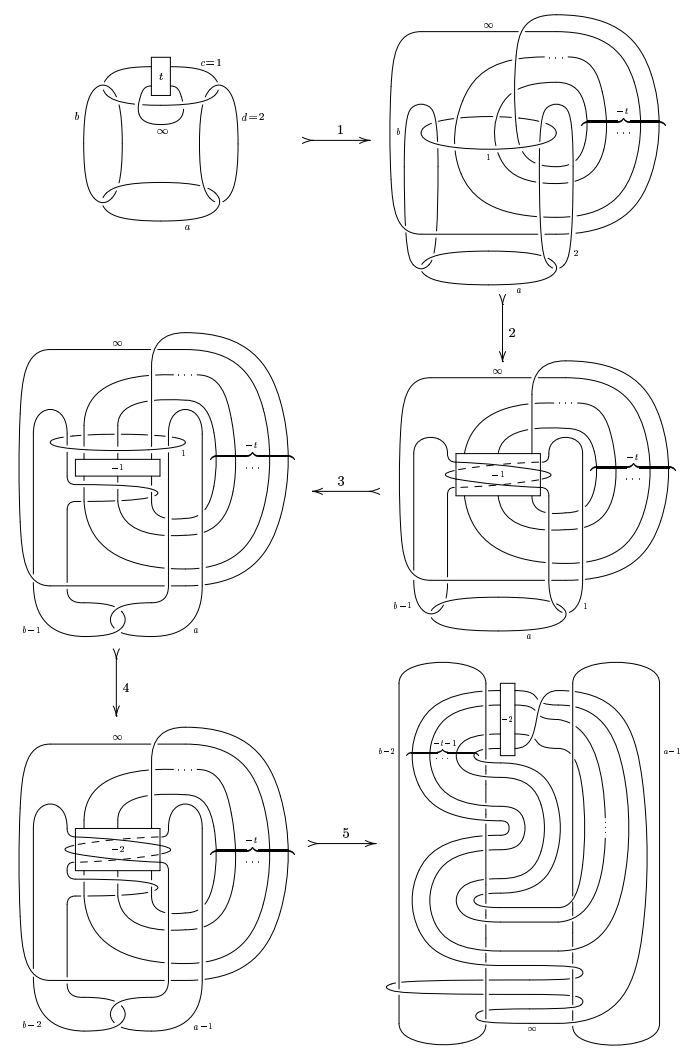

Figure 7. The equivalence of $M_{(a, b, 1,2)}^{t}(\infty)$ and $L(a-1,1) \# L(b-2,1)$ with $t<0$. 
In our third claim, we consider the manifolds for which $c=2$ and $d=1$.

Claim 3. $M_{(a, b, 2,1)}^{t}(\infty) \cong L(a-2,1) \# L(b-1,1)$.

Proof. There are two cases, $t>0$ and $t<0$, in the proof of this claim. The two cases are nearly identical. We refer the reader to Figures 4 and 5. In transition 1 , we perform a single negative twist about component $D$ giving it a trivial surgery coefficient. Component $D$ thus is removed from the diagram. The surgery coefficients of components $A$ and $C$ are each reduced by 1. We also see in transitions 1 and 2 a rather involved isotopy of component $K$ in which the twisting of components $K$ and $C$ is replaced by the looping of $K$ around $C$. This is done to facilitate transition 3, where we perform a single negative twist about component $C$. This gives $C$ a trivial surgery coefficient; so it is removed from the diagram. The surgery coefficients of components $A$ and $B$ are reduced to $a-2$ and $b-1$, respectively. Note that $K$ keeps its trivial surgery coefficient.

Transition 4 is the start of another involved isotopy. This isotopy separates components $A$ and $B$. The reader should first isotop the foremost (and leftmost) arc of component $A$ so that it moves: In front of the diagram, through the interior of the disk bounded by component $B$, and behind the diagram. During this isotopy, $A$ will snag one of the strands of component $K$. In transition 5 , we deform component $B$ to an oval. We note that components $A$ and $B$ are unlinked and unknotted. Thus each component, $A$ and $B$, represents a lens space summand of $M_{(a, b, 2,1)}^{t}(\infty)$ proving the claim.

Finally, we consider the manifolds for which $c=1$ and $d=2$.

Claim 4. $M_{(a, b, 1,2)}^{t}(\infty) \cong L(a-1,1) \# L(b-2,1)$.

Proof. Again there are two nearly identical cases to consider, $t>0$ and $t<0$. We refer the reader to Figures 6 and 7 . This proof is similar to that of Claim 3. Transition 1 is an isotopy of component $K$ in which the twisting of $K$ and $C$ is replaced by the looping of $K$ around $C$. This is done to facilitate transition 2 , where we perform a single negative twist about component $C$. This gives $C$ a trivial surgery coefficient; so it is removed from the diagram. The surgery coefficients of components $B$ and $D$ are each reduced by one.

Transition 3 is an isotopy which shrinks component $D$ and stretches component $A$. In transition 4 , we perform a single negative twist about component $D$ giving it a trivial surgery coefficient, and so it is removed from the diagram. The surgery coefficients of components $A$ and $B$ are reduced to $a-1$ and $b-2$, respectively.

We separate components $A$ and $B$ with an isotopy in transition 5 . The reader should refer to the transitions 4 and 5 in the proof of Claim 3 for 
clarification. We note that components $A$ and $B$ are unlinked and unknotted. Thus each component, $A$ and $B$, represents a lens space summand of $M_{(a, b, 1,2)}^{t}(\infty)$ proving the claim.

These four claims give us the following corollary. Part (a) of the corollary follows from Claims 2 and 3. Part (c) follows from Claims 1 and 4. And Part (b) follows from either Claims 2 and 4 or from Claims 1 and 3.

Corollary 2.1. If $t$ is an integer, then
a) $M_{(-2,-1,2,1)}^{t}(\infty) \cong L(-4,1) \# L(-2,1)$ and
$M_{(-2,-1,2,1)}^{t}(t) \cong L\left(t^{2}-2 t+1, t-2\right) \# L(2,1)$.
b) $M_{(-2,-1,1,2)}^{t}(\infty) \cong L(-3,1) \# L(-3,1)$ and
$M_{(-2,-1,1,2)}^{t}(t) \cong L\left(t^{2}-t+1, t-1\right) \# L(3,1)$, also
$M_{(-1,-2,2,1)}^{t}(\infty) \cong L(-3,1) \# L(-3,1)$ and
$M_{(-1,-2,2,1)}^{t}(t) \cong L\left(t^{2}-t+1, t-1\right) \# L(3,1)$,
c) $M_{(-1,-2,1,2)}^{t}(\infty) \cong L(-2,1) \# L(-4,1)$ and
$M_{(-1,-2,1,2)}^{t}(t) \cong L\left(t^{2}+1, t\right) \# L(4,1)$.

For the remainder of this paper, we wish to restrict our results to the cases which yield true lens spaces (thus excluding $S^{3}$ and $S^{2} \times S^{1}$ ). In Case (a), we must exclude the values $t=0,1$ and 2 . Likewise, in Case (b) we exclude $t=0$ and 1 , and in Case (c) we exclude $t=0$. All other integral values of $t$ are admissible.

We should remark, at this point, that these excluded cases are genuinely uninteresting. If the manifolds, before Dehn filling, were hyperbolic, then we might be able to claim a counterexample to a generalized cabling conjecture. But alas, the excluded manifolds are not hyperbolic.

\section{Hyperbolicity.}

Let $M$ be any one of the manifolds $M_{(-1,-2,1,2)}^{t}, M_{(-1,-2,2,1)}^{t}, M_{(-2,-1,1,2)}^{t}$ and $M_{(-2,-1,2,1)}^{t}$ where $t$ is an admissible integer. In this section, we prove that $M$ is hyperbolic (i.e., the interior of $M$ admits a hyperbolic structure). Since each manifold $M$ has toroidal boundary $T=\partial M$, we need to show $M$ is irreducible, $\partial$-irreducible, atoroidal, and not Seifert fibered $[\mathbf{T}]$.

Lemma 3.1. If $S$ is a separating reducing 2-sphere in $M(\infty)$ or $M(t)$, then $S$ may not be isotoped so that $S \cap T=\emptyset$.

Proof. It suffices to prove the claim for $M(t)$ since if a reducing 2-sphere $S$ could be made disjoint from $T$ in $M(\infty)$, then either $S$ would also be a reducing 2-sphere disjoint from $T$ in $M(t)$ or $S$ would be inessential in $M(t)$. In the latter case, both summands of $M(t)$ would have to appear in $M(\infty)$. 
First note that, for the connect sum of two irreducible manifolds, there is only one isotopy class of reducing (essential) 2-spheres. By Corollary 2.1, $M(t)$ is homeomorphic to the connect sum of two lens spaces. Thus any reducing 2-sphere in $M(t)$ can be isotoped to a "standard" sphere $S$ which misses the cores of the lens space summands. That is, we may assume $S$ separates to the two link components with the nontrivial surgery coefficients shown in the final diagrams in Figures 2 and 3. Since $S$ is reducing, it must separate the two cores and intersect the component $K$. Recall that $T$ is the torus boundary of a regular neighborhood of $K$. Now the problem can be restated by claiming $K$ cannot be isotoped in $M(t)$ to miss $S$.

Again, we refer the reader to the final diagrams in Figures 2 and 3. Note that the link diagrams are identical, only the surgery coefficients differ. For this link diagram, with $t \neq 0$, the Alexander polynomial is given by $\Delta(a)=a^{2}-4 a+6-4 a^{-1}+a^{-2}$ when all components are given a clockwise orientation. Recall that if $L$ is a split link, then the Alexander polynomial is zero for that link. So we conclude that the three components in this diagram are indeed linked.

Every arc of $K$ in $M(t)-S$ links with the core of the lens space summands. So for any product neighborhood $S \times I$ of $S$, where $S=S \times\{0\}$, such that $S \times\{1\}$ and $K$ intersect transversaly, we have $|(S \times\{1\}) \cap K| \geq|S \cap K|$. Therefore, from Proposition 1.1 of $[\mathbf{E}],|S \cap K|$ is minimal. So, it is impossible for $K$ to be isotoped to miss $S$.

Lemma 3.2. $M$ is irreducible and $\partial$-irreducible.

Proof. Suppose that $M$ is reducible with $S$ a reducing sphere in $M$. If $S$ is nonseparating in $M$, then $S$ is nonseparating in $M(r)$. But by Corollary 2.1, there are slopes $r$ for which $M(r)$ is the connect sum of two lens spaces. And these manifolds contain no nonseparating spheres.

So assume that $S$ is a separating sphere in $M$. Then $M=X \# Y$ where $\partial X=\partial M$ and $Y \neq S^{3}$. Thus $M(r)=X(r) \# Y$. In particular, $M(\infty)=$ $X(\infty) \# Y \cong L_{1} \# L_{2}$ where $L_{1}$ and $L_{2}$ are lens spaces. By the uniqueness of decomposition, we can assume $X(\infty) \cong L_{1}$ and $Y \cong L_{2}$. But this contradicts Lemma 3.1.

If $M$ is $\partial$-reducible, since $M$ is irreducible and $\partial M$ is a torus, then $M$ must be a solid torus. But this is impossible since the fillings of a solid torus are well-known and do not correspond to the results of Corollary 2.1.

Bireducible manifolds are not Seifert fibered. Thus we have the following lemma:

\section{Lemma 3.3. $M$ is not Seifert fibered.}

Proof. If $M$ is Seifert fibered, then $M(r)$ is Seifert fibered for all but one slope $r$ for which $M$ is reducible [H]. But by Corollary 2.1, we have two slopes for which Dehn filling produces reducible manifolds. 
The following lemma is proved in $[\mathbf{E W}]$ :

Lemma 3.4 (Eudave-Muñoz, Wu). Let $W$ be an irreducible and $\partial$-irreducible 3-manifold. If both $W\left(r_{1}\right)$ and $W\left(r_{2}\right)$ are reducible and $\partial$-reducible, then $r_{1}=r_{2}$.

Next, we show that $M$ does not contain an essential torus.

Lemma 3.5. $M$ is atoroidal.

Proof. Suppose that $M$ contains an essential torus $F$. Then $F$ must be separating. Otherwise, $M(\infty)$ would contain a nonseparating torus or sphere, contradicting Corollary 2.1. Let $W$ and $W^{\prime}$ be the two components of $M$ cut along $F$, where $W$ contains $\partial M$. Using Lemma 3.2, we may conclude that $W$ is both irreducible and $\partial$-irreducible (as is $W^{\prime}$ ). By Corollary 2.1, both $M(\infty)$ and $M(t)$ are atoroidal and reducible. Thus $F$ must be compressible in both $W(\infty)$ and $W(t)$.

If both $W(\infty)$ and $W(t)$ are reducible, then this contradicts Lemma 3.4. Thus, we may assume that one of them is irreducible (i.e., it is a solid torus).

Let $x \in\{\infty, t\}$ such that $W(x) \cong S^{1} \times D^{2}$. Let $y \in\{\infty, t\}$ and $x \neq y$. Let $K_{x}$ be the core of the Dehn filling, and $V_{x}=N\left(K_{x}\right)$. Then $W=$ $W(x)-\operatorname{int} V_{x}$. Note that $W^{\prime}=M-W$ and $W^{\prime}=M(\gamma)-W(\gamma)$, for all slopes $\gamma \in \partial M$. Recall that $F=\partial W^{\prime} \cong \partial W(\gamma)$. Therefore $W^{\prime}=$ $M(x)-W(x) \cong(L(a, b) \# L(p, q))-S^{1} \times D^{2}$. Let $r$ be a slope in $F$ which corresponds to a meridian of $W(x) \cong S^{1} \times D^{2}$. Then $W^{\prime}(r)$ is reducible.

Now we examine $W^{\prime}=M(y)-W(y)$. Let $s$ be the slope in $F$, which corresponds to a meridian in $\partial W(y)$. So, $r$ is the meridian slope in $\partial W(x)$ and $s$ is the slope of the new meridian in $\partial W(y) \cong \partial W(x)$ after performing surgery on $K_{x}$ along $y$.

We consider two cases, according to whether $W(y)$ is reducible or not. Case 1: $W(y)$ is reducible (i.e., $W(y) \cong S^{1} \times D^{2} \# L(p, q)$ ).

It follows from theorems of Gabai and Scharlemann $([\mathbf{G a}]$ and $[\mathbf{S}])$ that $W$ is a cable space.

Case 2: $W(y)$ is irreducible.

From Gabai's Theorem $1.1[\mathbf{G a}], W$ is the exterior of a braid in a solid torus. So, in both cases, we can apply Gordon's Lemma 3.3 [Go] (here $W$ and $K_{x}$ take place of respectively $Y$ and $\left.J\right)$. Thus, $\Delta(r, s)=\left|\frac{n w^{2}}{(w, m)}\right| \geq|w|$, where $w$ is the winding number of $K_{x}$ in the solid torus $W(x)$.

In the first case, $K_{x}$ is $(p, q)$-cable knot (hence $q \geq 2$ ). So $|w|>1$. In the second case, $K_{x}$ is a braid, then again $|w|>1$. Consequently, $\Delta(r, s)>1$. Note that this result also follows from $[\mathbf{B}]$ Theorem 2.5.

Now, in the first case $W^{\prime}(s)$ is a lens space, which contradicts [BZ]. In the second case, $W^{\prime}(s)$ is also reducible, which contradicts [GLu]. 
The results of this section show that any one of the manifolds $M_{(-1,-2,1,2)}^{t}$, $M_{(-1,-2,2,1)}^{t}, M_{(-2,-1,1,2)}^{t}$ and $M_{(-2,-1,2,1)}^{t}$, where $t$ is an admissible integer, is a hyperbolic manifold. This fact and Corollary 2.1 suffice to prove Theorem 1.

\section{Comments and questions.}

The use of both positive and negative values in the parameter $t$ produces redundancy in the list of manifolds up to homeomorphism. This redundancy is made explicit in the correspondences shown in the next theorem.

Theorem 2. The following manifolds are homeomorphic:

a) $M_{(-2,-1,2,1)}^{t}(t) \cong M_{(-2,-1,2,1)}^{2-t}(2-t)$ for all $t \leq-1$.

b) $M_{(-1,-2,2,1)}^{t}(t) \cong M_{(-1,-2,2,1)}^{1-t}(1-t)$ for all $t \leq-1$.

c) $M_{(-1,-2,1,2)}^{t}(t) \cong M_{(-1,-2,1,2)}^{-t}(-t)$ for all $t \leq-1$.

Proof. This proof is based on the fact that two lens spaces $L(p, q)$ and $L\left(p, q^{\prime}\right)$ are of the same homeomorphism type if and only if $\pm q q^{\prime} \cong 1(\bmod p)[\mathbf{R}]$. We only prove Case (a), as the other two cases are similar. By Corollary 2.1, $M_{(-2,-1,2,1)}^{t}(t) \cong L\left(t^{2}-2 t+1, t-2\right)$ and $M_{(-2,-1,2,1)}^{2-t}(2-t) \cong L\left(t^{2}-2 t+1,-t\right)$. The homeomorphism of the two follows since $-(2-t)(-t)=2 t-t^{2}=$ $1-\left(t^{2}-2 t+1\right)$.

We would like to point out that all known examples of bireducible fillings result in a summand which is homeomorphic to one of the lens spaces $L(2,1), L(3,1)$, or $L(4,1)$. The Eudave-Muñoz and $\mathrm{Wu}$ examples $[\mathbf{E W}]$ and the Gordon and Litherland example [GLi] always have an $L(2,1)$ summand. This begs the question: Does there exist an example in which no summand is homeomorphic to either $L(2,1), L(3,1)$, or $L(4,1)$ ?

Second, we would like the reader to note that there are no known examples of "trireducible" manifolds. According to Gordon and Luecke [GLu], if two fillings on an irreducible manifold with torus boundary produce reducible manifolds, then the slopes of the fillings must have a minimal geometric intersection of one. This means an irreducible manifold can have at most three slopes for which Dehn filling produces a reducible manifold. Does there exist a manifold which is trireducible?

Finally, in all known examples, the minimum number of times one of the reducing spheres meets the core of the Dehn filling is bounded by four. In our examples and the Eudave-Muñoz and $\mathrm{Wu}$ examples $[\mathbf{E W}]$, the other reducing sphere in each family meets the core an arbitrarily large number of times. Does there exist an example in which both minimal intersections are larger than four? And if so, is there a family of examples in which both minimal intersections are unbounded? 
For the readers' convenience, here is a way to construct examples of hyperbolic manifolds with a single boundary manifold which have bireducible Dehn fillings, from the examples given in $[\mathbf{E W}]$.

It is shown in [EW] Theorem 3.6 that there is a collection of hyperbolic manifolds, denoted $M_{p}$, with two toroidal boundary components $T_{0}$ and $T_{1}$, such that $T_{0}$ has two reducing slopes. If $r$ is a slope in $T_{0}$, denote the Dehn filling along $r$ by $M_{p}(r)$; and, if $r$ is in $T_{1}$, denote the Dehn filling by $(r) M_{p}$.

It follows from [EW] Lemma 3.1 that for the slopes $\infty$ and 0 in $T_{0}$, both $M_{p}(\infty)$ and $M_{p}(0)$ are reducible. Now, from [EW] Figure 3.1, $(\infty) M_{p}$ and (0) $M_{p}$ are also both reducible, where $\infty$ and 0 are slopes in $T_{1}$. Then, from [EW] Table 1.1, if $r$ is a slope in $T_{1}$ such that $\Delta(r, \infty)>3$ or $\Delta(r, 0)>3$ then $(r) M_{p}$ is hyperbolic.

So, for almost all $r$ (for all except at most 16 values of $r$ ) $(r) M_{p}$ is a hyperbolic manifold, with a single boundary component, and with bireducible Dehn fillings. Furthermore, $(r) M_{p}(\infty)=\mathrm{R} P^{3} \# S_{1}$ and $(r) M_{p}(0)=$ $\mathrm{R} P^{3} \# S_{2}$, where $S_{1}$ and $S_{2}$ are small Seifert fibered spaces (in a few cases they are lens spaces).

Acknowledgements. We would very much like to thank the referee for some very insightful comments and Jeff Weeks and A.C. Manoharan for SnapPea PC which helped tremendously in verifying our work.

\section{References}

[B] J. Berge, The knots in $D^{2} \times S^{1}$ which have nontrivial Dehn surgeries that yield $D^{2} \times S^{1}$, Topology Appl., 38 (1991), 1-19, MR 92d:57005, Zbl 0725.57001.

[BZ] S. Boyer and X. Zhang, On Culler-Shalen seminorms and Dehn filling, Annals of Math., 148 (1998), 1-66, MR 2000d:57028.

[E] M. Eudave-Muñoz, 4-Punctured tori in the exterior of knots, J. Knot Theory Ramifications, 6 (1997), 659-676, MR 98f:57022, Zbl 0887.57014.

[EW] M. Eudave-Muñoz and Y.-Q. Wu, Nonhyperbolic Dehn fillings on hyperbolic 3manifolds, Pacific J. Math., 190 (1999), 261-275, MR 2000j:57035.

[Ga] D. Gabai, Surgery on knots in solid tori, Topology, 28 (1989), 1-6, MR 90h:57005, Zbl 0678.57004.

[GS] F. González-Acuña and H. Short, Knot surgery and primeness, Math. Proc. Camb. Phil. Soc., 99 (1986), 89-102, MR 87c:57003, Zbl 0591.57002.

[Go] C. McA. Gordon, Dehn surgery and satellite knots, Trans. Amer. Math. Soc., 275 (1983), 687-708, MR 84d:57003, Zbl 0519.57005.

[GLi] C. McA. Gordon and R.A. Litherland, Incompressible planar surfaces in 3manifolds, Topology Appl., 18 (1984), 121-144, MR 86e:57013, Zbl 0554.57010.

[GLu] C. McA. Gordon and J. Luecke, Reducible manifolds and Dehn surgery, Topology, 35 (1996), 385-409, MR 97b:57013, Zbl 0859.57016.

[H] Heil, Elementary surgery on Seifert fiber spaces, Yokohama Math. J., 22 (1974), 135-139, MR 51 \#11515, Zbl 0297.57006. 
[R] D. Rolfsen, Knots and Links, Math. Lect. Ser., 7, Publish or Perish, Berkeley, California, 1976, MR 58 \#24236, Zbl 0339.55004.

[S] M. Scharlemann, Producing reducible 3-manifolds by surgery on a knot, Topology, 29 (1990), 481-500, MR 91i:57003, Zbl 0727.57015.

[T] W. Thurston, Three dimensional manifolds, Kleinian groups and hyperbolic geometry, Bull. Amer. Math. Soc., 6 (1982), 357-381, MR 83h:57019, Zbl 0496.57005.

Received April 18, 2000 and revised February 23, 2002.

Analytical Mechanics Associates Inc.

303 Butler FARM RoAd

HAMPTON VA 23666

E-mail address: hoffman@ama-inc.com

Université de Provence

C.M.I. 39

Rue Joliot Curie

F-13453 Marseille Cedex 13

FRANCE

E-mail address: matignon@gyptis.univ-mrs.fr 


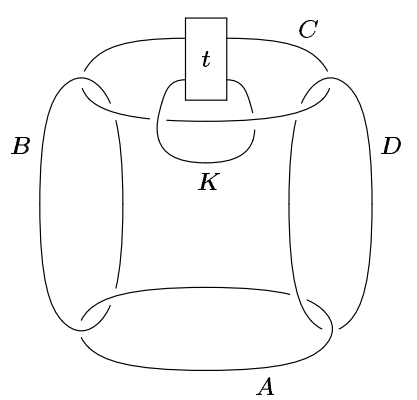

